\title{
Identification of key pathways and genes in PTEN mutation prostate cancer by bioinformatics analysis
}

\author{
Jian Sun ${ }^{\dagger}$, Shugen Li ${ }^{\dagger}$, Fei Wang, Caibin Fan and Jianqing Wang ${ }^{*}$ (B)
}

\begin{abstract}
Background: Prostate cancer (Pca) remains one of the leading adult malignancies. PTEN (Phosphatase and Tensin Homolog) mutant is the top common mutated genes in prostate cancer, which makes it a promising biomarker in future individualized treatment.

Methods: We obtained gene expression data of prostate cancer from TCGA (The Cancer Genome Atlas) database for analysis. We analyzed the DEGs (differentially expressed genes), and used online tools or software to analyze Gene ontology (GO) and the Kyoto Encyclopedia of Genes and Genomes (KEGG), Gene set enrichment analysis (GSEA), Search Tool for the Retrieval of Interacting Genes/Proteins, and Molecular Complex Detection.

Results: Latest TCGA data showed PTEN mutation in about 22\% patients. 1736 DEGs in total were identified. Results of gene functional enrichment analyses showed that muscle contraction, negative regulation of growth and multiple metabolic progression were significantly enriched. GNG13, ACTN2, POTEE, ACTA1, MYH6, MYH3, MYH7, MYL1, TNNC1 and TNNC2 were the top ten hub genes. Patients with PTEN mutation showed relatively decreased mRNA expression level of PTEN. Survival analysis indicated the risk of disease recurrence in patients with PTEN mutation.

Conclusions: Our findings suggested that PTEN mutation in prostate cancer may induce changes in a variety of genes and pathways and affect disease progression, suggesting the significance of PTEN mutation in individualized treatment of prostate cancer.
\end{abstract}

Keywords: Prostate cancer, Bioinformatics analysis, PTEN mutation, TCGA, RNA seq

\section{Background}

Prostate cancer is one of the common malignant tumors in urology and is the leading cause of cancer-related mortality in men in developed countries. [1]. Previous studies have focused on signaling pathways, critical oncogenes and related cellular processes that promote disease progression, but the underlying molecular biologic mechanisms are still not clear. To further explore some new mechanisms or treatment targets, various online databases, such as The Cancer Genome Atlas (TCGA) and Gene Expression Omnibus (GEO), offer gene expression data and correlated variable clinical course of mutiple

\footnotetext{
* Correspondence: jqwang14@fudan.edu.cn

${ }^{\dagger}$ Jian Sun and Shugen Li co-first contributors for this work

Department of Urology, The Affiliated Suzhou Hospital of Nanjing Medical

University, 26 Daoqian Rd, Suzhou 215000, Jiangsu, China
}

cancer types [2]. However, until now, there have been few researches focused on the individualized treatment based on the datasets above.

Gene mutations have been shown to be critical in disease program. In primary prostate cancer, metastatic prostate cancer, and metastatic, castration-resistant prostate cancer (mCRPC), recurrent somatic mutations, copy number changes, and oncogenic structural DNA rearrangements (chromosomal abnormalities) have been identified [2-5]. The combined results of several studies have confirmed that PTEN is the top common mutated genes in $\mathrm{PCa}$, indicating its potential clinical significance. PTEN is a multi-functional tumor suppressor and, loss of expression of PTEN has been found in about $70 \%$ of PCa patients [6, 7]. Functionally, PTEN regulates cell proliferation and survival mainly by regulating PI3K-

(c) The Author(s). 2019 Open Access This article is distributed under the terms of the Creative Commons Attribution 4.0 International License (http://creativecommons.org/licenses/by/4.0/), which permits unrestricted use, distribution, and 
AKT related signaling pathways [8]. Recent years, latest researches indicated that PTEN could modulate cell growth via AKT-independent mechanisms [9]. Moreover, prostate-specific Pten deletion in mice model results in significantly shortened course of prostatic intraepithelial neoplasia development and promotes metastatic prostate cancer formation [6].

In prostate cancer, PTEN is commonly altered through copy-number loss rather than point mutation. Previous researches have focused on PTEN mRNA expression in prostate cancer initiation and development [10]. Since the PTEN mutation is quite common in prostate cancer patients, we want to know whether the cluster analysis of the tumor molecular spectrum data will define the PTEN mutant tumor as a subgroup. In addition, various critical cell signaling pathways and processes may change in patients with PTEN mutations compared to PTEN wild-type patients [8]. Therefore, different PTEN status may influence disease development, outcome and therapeutic strategies, causing naturally resistant. Further study of the changes in cell processes and pathways in patients with PTEN mutations will contribute to further understanding of the pathogenesis of the disease and provide more novel evidence for the accurate treatment of prostate cancer.

In the present study, we used the data of a RNA sequencing (RNA-Seq) dataset to identify the mechanims underlying PTEN mutation in prostate cancer by bioinformatics analysis, expecting to reveal the potential function of PTEN mutation in predicting prognosis and individualized treatment options.

\section{Methods}

Data information

We obtained the data and clinical profiles of a prostate cancer RNA-Seq (project: TCGA-PRAD) from TCGA
(The Cancer Genome Atlas) database. Other related clinical resourses were downloaded directly from the cBioPortal website (website: http://www.cbioportal.org) [11].

\section{Analysis of GSEA (gene set enrichment analysis)}

To understand the effect of PTEN mutation on various biological function gene sets in prostate cancer patients, GSEA was used to do the enrichment analysis. The number of permutations was set at 5 . A $P$-value cutoff of 0.05 with a false discovery rate (FDR q-val) $<0.25$ were considered statistically significant $[12,13]$. GSEA v3.0 (http://software.broadinstitute.org/gsea/downloads.jsp).

\section{Identification of differentially expressed genes (DEGs)}

We used EdgeR to study gene differential expression of mRNA levels between PTEN mutations and PTEN wildtype prostate cancer patients $[14,15]$. Genes with |fold change (FC) $\mid \geq 2$ and both the $P$-value and FDR $<0.05$ were identified as DEGs.

\section{Functional enrichment analysis}

Candidate DEGs functions and pathways enrichment were analyzed using $\mathrm{R}$ package clusterProfiler. Gene ontology (GO) analysis and pathway analysis were carried out using Gene Ontology website (Available online: http://www.geneontology.org/) and KEGG PATHWAY (Available online: http://www.genome.jp/kegg) with a $P$ value cutoff of $<0.05$.

\section{Protein-protein interaction analysis}

We used Search Tool for the Retrieval of Interacting Genes (STRING) database to analyze the protein interation network [16]. We submitted the DEGs to the website to evaluate the interactive relationships. Combined score $>0.4$ were selected as significant. Then, the module screening was completed by

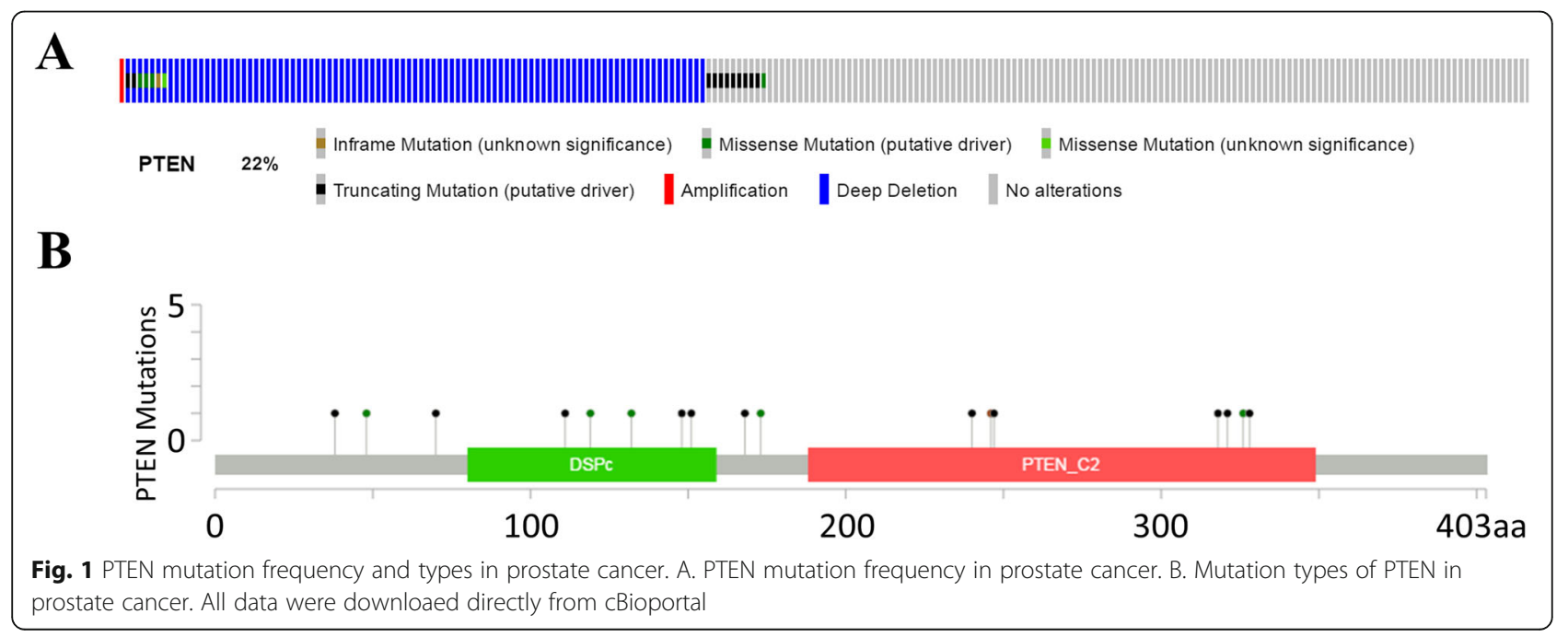


Molecular Complex Detection (MCODE) in Cytoscape (scores $>3$ and nodes $>4$ ) [17].

\section{Statistical analyses}

Student's t-test was used to compare the PTEN mRNA expression level and Gleason score between PTEN mutation and PTEN wild-type prostate cancer tissue. We evaluated the prognosis between different PTEN status groups using the Kaplan-Meier method with log-rank test by Graphpad. A value of $\mathrm{P}<0.05$ was considered statistically significant. All the statistical analyses were conducted with Graphpad and $\mathrm{R}$ 3.3.0 as previously [18].

\section{Results}

\section{Data information}

We downloaded the gene expression data and clinical information of 499 prostate cancer patients from TCGA

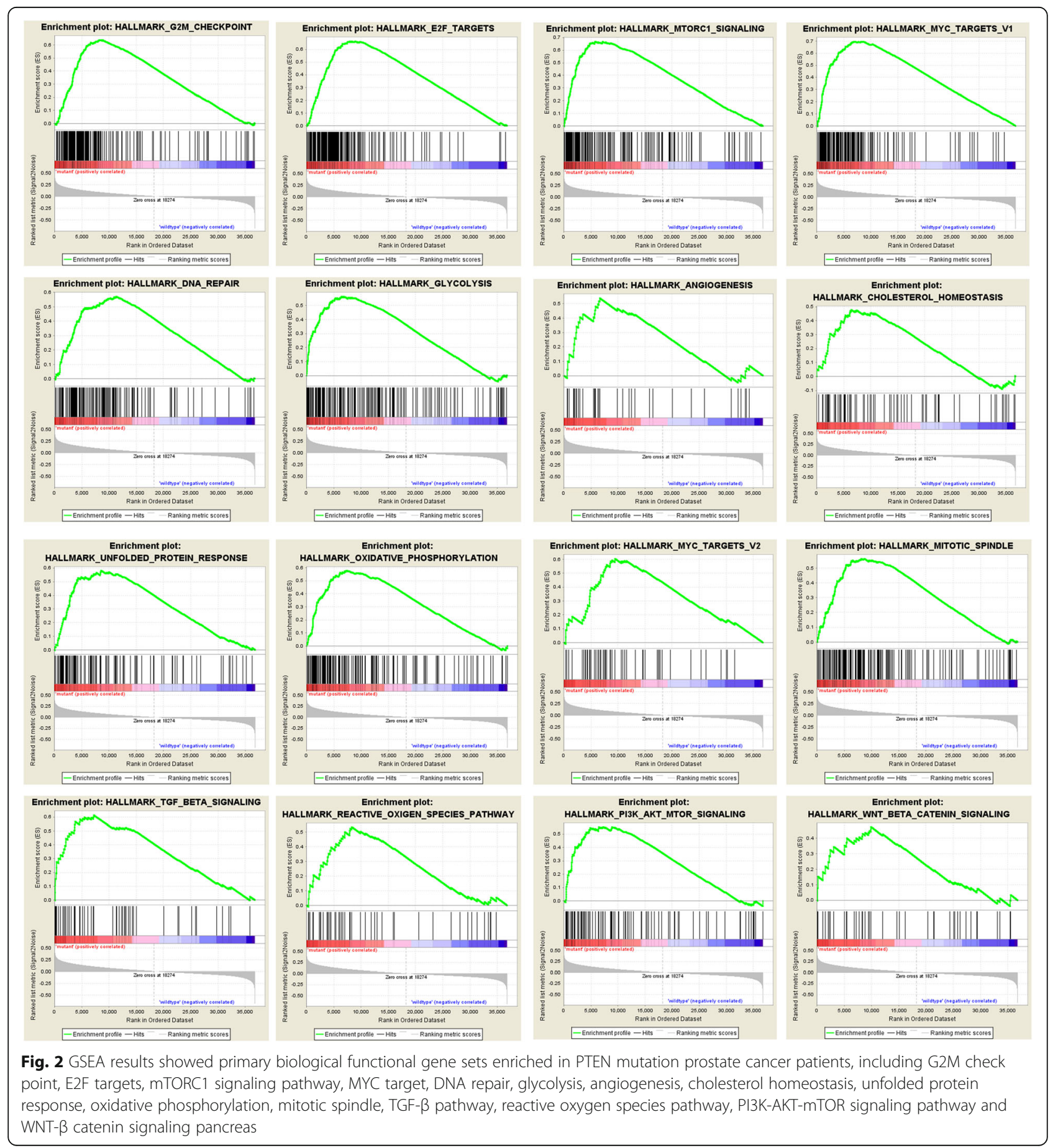


database (project: TCGA-PRAD). 108 patients (22\%) were with PTEN mutation and the rest were PTEN wild type (Fig. 1 a). Mutation types included deep deletion, truncating, and missense mutations spanning over entire gene (Fig. 1 b).

\section{GSEA}

To further explore the function of PTEN mutation in disease progression, we analyzed the a variety of biological functional gene sets by the GSEA. As in Fig. 2, results showed that biological processes including G2M check point, DNA repair, glycolysis, angiogenesis, cholesterol homeostasis, unfolded protein response and oxidative phosphorylation were significantly enriched. This suggests that PTEN mutation might infuence disease program by regulating mitosis, DNA repair and metabolism. E2F targets and MYC targets were significantly enriched in GO enrichment analysis. Furthermore, enriched functional pathways included mTORC1 signaling pathway, PI3K-AKT-mTOR signaling pathway, WNT- $\beta$ - catenin signaling, reactive oxigen species pathway and TGF- $\beta$ pathway (Fig. 2 ).

\section{Clinical affairs of PTEN mutation}

We then focused on the clinical impact of PTEN mutation on prostate cancer. We first explored the PTEN mRNA expression level in both group and found that PTEN was downregulated in tumor tissues with PTEN mutation (Fig. 3 A). Meanwhile, patients with PTEN mutation showed relatively higher Gleason score, which determines the histological grading of prostate cancer (Fig. 3 B). Furthermore, we did Kaplan-Meier analysis to evaluate whether PTEN mutation correlates with and prostate cancer recurrence. Data from cBioportal

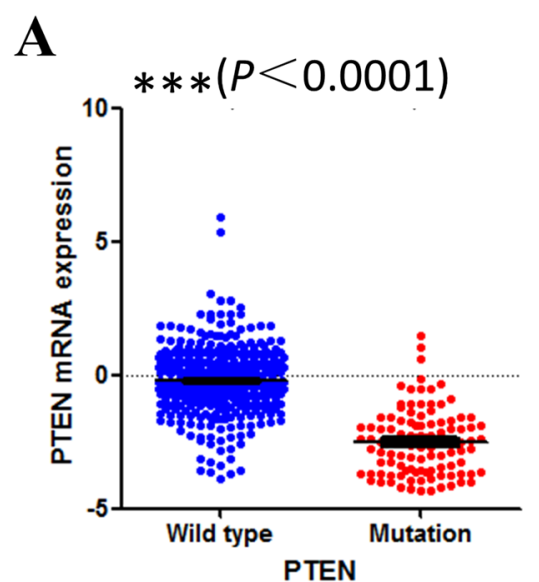

$\mathcal{C}$
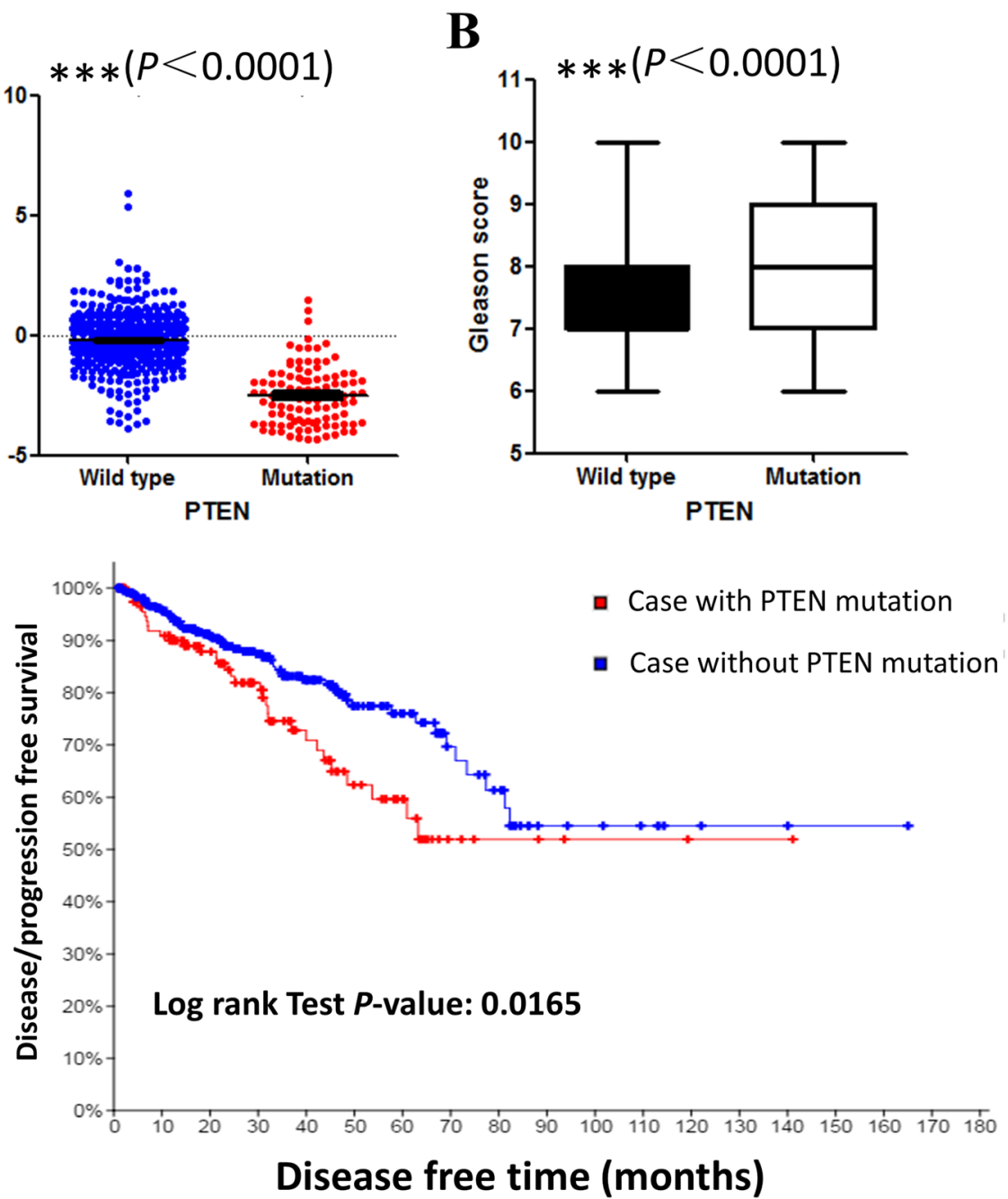

Fig. 3 Mutations of PTEN is associated with lower PTEN expression level, higher Gleason score and poorer prostate cancer prognosis. A. Correlation between PTEN mutation and PTEN mRNA expression. B. Correlation between PTEN mutation and Gleason score. C. Survival curves of PCa patients stratified by PTEN mutation 
confirmed that patients with PTEN mutation often have relatively poorer prognosis than those without mutation (Fig. $3 \mathrm{C}$ ). All results above indicated the significance of PTEN mutation in prostate cancer clinical impact.

\section{Identification of DEGs}

To further explore the underlying mechanisms by which PTEN mutation influences prostate cancer progression, we identified the DEGs. A total of 1736 genes were identified as DEGs, of which, 344 were upregulated and 1392 were downregulated. Figure 4 shows the volcano plot of the DEGs, while the heat map of top 100 DEGs is shown in Additional file 1: Fig. S1.

\section{GO and KEGG analysis of DEGs}

To further analyze the DEGs at the functional level, we used $R$ package cluster Profiler for our GO/KEGG enrichment analysis to deal with the 1736 DEGs. The GO analysis (Fig. 5 A) suggested significant enrichment in muscle contraction, muscle filament sliding, actinmyosin filament sliding, muscle system process, actinmediated cell contraction, striated muscle contraction, actin filament-based movement and myofibril assembly.

Furthermore, results of KEGG pathway analysis showed significant enrichment in olfactory transduction, cardiac muscle contraction, hypertrophic cardiomyopathy (HCM), neuroactive ligand-receptor interaction, adrenergic signaling in cardiomyocytes, dilated cardiomyopathy (DCM), salivary secretion and mineral absorption (Fig. 5 B).
Protein-protein interaction analysis and module screening We then uploaded all DEGs above to the STRING database to investigate the protein interaction and identify critical hub genes. We found out the top ten genes as hub genes which were ranked by degree. The top 10 hub genes were GNG13, ACTN2, POTEE, ACTA1, MYH6, MYH3, MYH7, MYL1, TNNC1 and TNNC2. GNG13 had the highest degree of nodes with 55. Then, we used the MCODE plugin in Cytoscape to identify the modules of genes in PPI network. Then we did functional enrichment analysis based on the top three significant modules (Additional file 2: Table S1 and Fig. 6, respectively). We selected the top 3 significant modules and did GO/ KEGG analysis. KEGG and GO analyses found enrichment only in module 1. KEGG annotation in module 1 genes was enriched in tight junction and cardiac muscle contraction. GO analysis showed that the genes in module 1 were mainly associated with muscle filament sliding, cardiac muscle contraction and ventricular cardiac muscle tissue morphogenesis.

\section{Discussion}

PTEN, which is a tumor suppressor protein and is very commonly lost across cancer types [19]. The major function of PTEN depends on its phosphatase activity. PTEN mainly inhibits PI3K/AKT pathway activity, while other studies also suggest that PTEN may function through AKT-independent [8]. Mutations in PTEN result in losing its phosphatase activity, which contribute to the

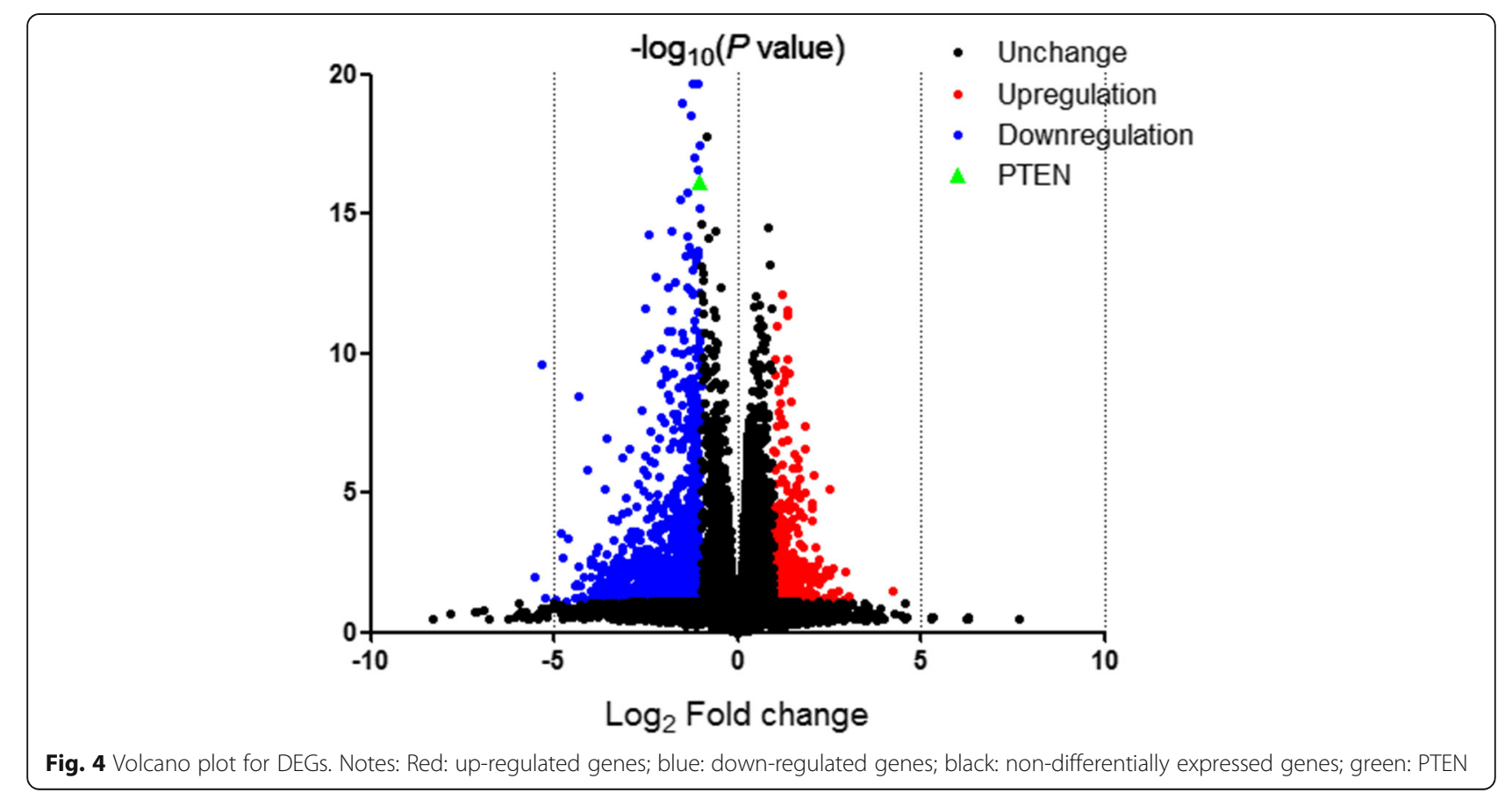




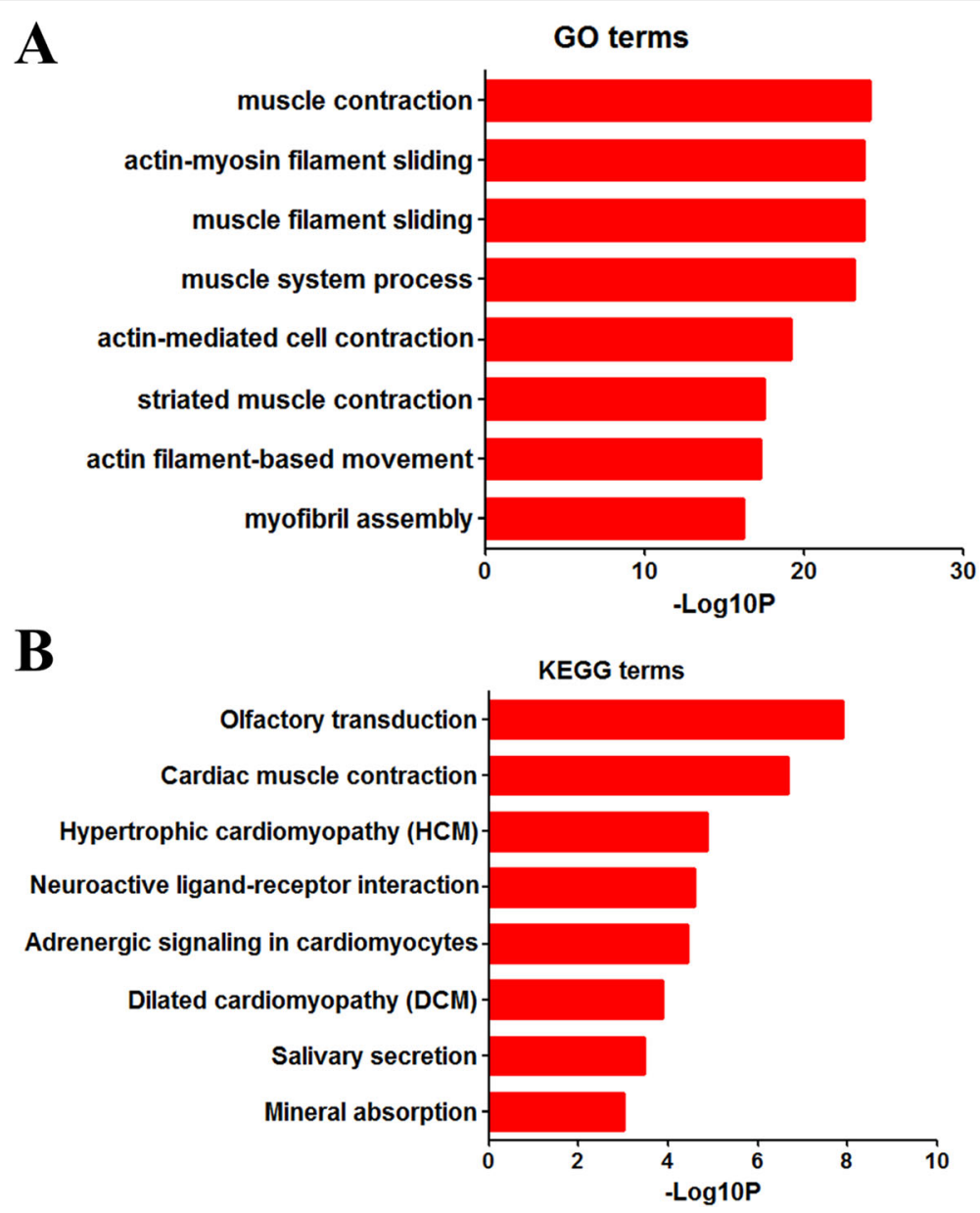

Fig. 5 Functional enrichment results of differentially expressed genes. A. Top 8 pathways identified in the GO enrichment analysis in DEGs. B. Top 8 pathways identified in KEGG pathway analysis in DEGs

onset and progression of tumors and related diseases, including hereditary cancers such as prostate cancer.

Here in our study, we analyzed the gene expression data of prostate cancer obtained from TCGA to uncover the critical pathways and top hub genes associated with PTEN mutation. We found $22 \%$ patients with PTEN mutation among all cases. The mRNA expression and clinical affair analyses showed lower expression level of PTEN, higher Gleason score and poorer prognosis in patients with PTEN mutation, which indicated the significance of PTEN mutation in prostate cancer: PTEN mutation correlated with advanced disease and worse outcome. PTEN deletion, transcriptional and epigenetic modifications of PTEN are known mechanisms that could cause deregulation of PTEN [20, 21]. Lower PTEN expression level often correlates to disease progression in various cancer types [22-24], which makes it one of the important potential mechanisms how PTEN mutation exert the role in disease progression. In the following research, the relationship between PTEN mutation and more details of clinical affairs of prostate cancer requires larger sample data for more accurate results.

Now that PTEN mutation contributes to prostate cancer progression, the mechanisms underlying other than PTEN downregulation are critical. GSEA analysis suggests that PTEN mutation were mainly associated with the cell metabolism, proliferation and cancer related pathways. In the cellular processes above, metabolic processes, including glycolysis and lipid metabolism, always play critical roles in cancer progression. Previous studies have shown that the formation of cancer cells requires adaptations across various metabolic processes to satisfy the energy required for their increased rate of proliferation. Dysregulation of lipid metabolism, including upregulation of several lipogenic enzymes, has been a hallmark of prostate cancer, and metabolic target has been shown to be a potential treatment target in prostate cancer $[25,26]$. Our results indicated that drugs targeting lipid metabolic pathways could contribute to the development of new therapeutic modalities in PTEN 


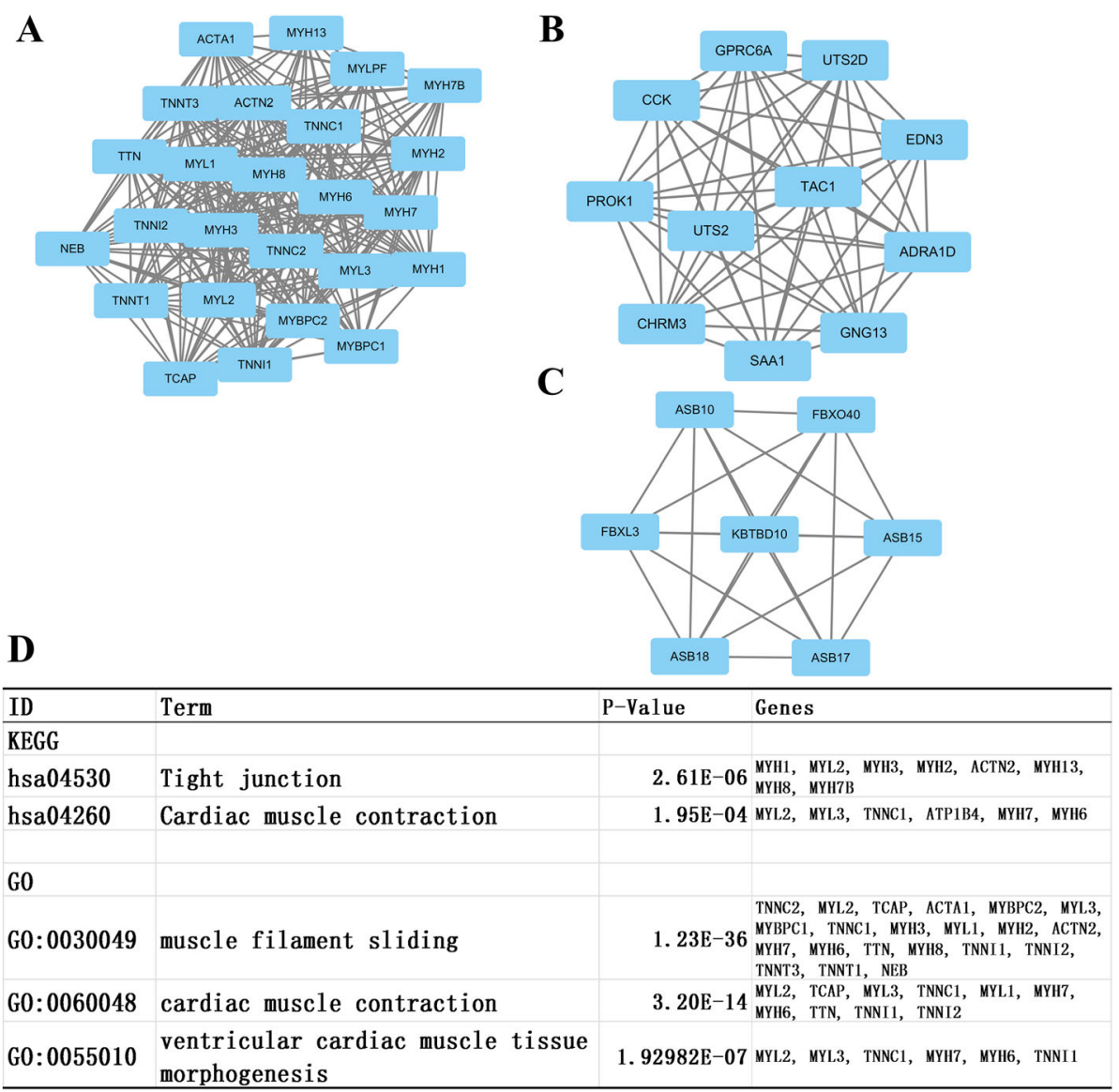

Fig. 6 Top three modules from the PPI network and the results of KEGG and GO analyses of module 1. A. Protein interaction network of module 1; B. Protein interaction network of module 2; C. Protein interaction network of module 3; D. GO and KEGG analyses of module 1

mutation prostate cancer patients. As for DNA repair, previous researches have suggested that MCRPC has genomic aberrations that implicated in DNA repair [27, 28]. Poly (adenosine diphosphate [ADP]-ribose) polymerase (PARP) is a critical factor that functions in the biologic progression of DNA repair, and PARP inhibition has been shown to exert anti-tumor activity in sporadic mCRPC cases by treating DNA repair defects in tumor cells [29]. In addition, antiandrogen therapy, the main strategy for the treatment of prostate cancer, could significantly lead to increased DNA damage and reduced colony formation survival. Our findings suggested that mechanisms implicated with DNA repair might contribute to disease progression, thus providing new insights into the development mechanisms of prostate cancer.

Today, the detection of mutations in important tumor genes and tumor suppressor genes has been applied clinically. These test results can help clinicians select more effective individualized treatment strategies. Our results indicate that $\mathrm{G} 2 \mathrm{M}$ check point is one of the biological processes significantly enriched in patients with PTEN mutation. As we all know, first-generation taxane chemotherapies, Docetaxel (Taxotere), which is the first-line drug in prostate cancer chemotherapy, effectively target the cytoskeleton by stabilizing the interaction of $\beta$-tubulin subunits of microtubules preventing depolymerization, inducing G2M arrest and apoptosis [30]. This suggests that patients with PTEN mutation may be more sensitive to Docetaxel. Moreover, our results above have shown that PTEN mutation correlated with advanced disease and worse outcome. Combining these results, we provide the evidence that early intervention is required for patients with PTEN mutation to obtain a longer survival period, and that chemotherapies using Docetaxel might be more effective in such patients, which provides a potential proof for individualized treatment.

We then identified the DEGs in both groups and carried out the functional analysis. 1736 genes in all were identified as DEGs with different PTEN mutation status. Enrichment analysis indicated that DEGs in PTEN mutation patients contributed to cell movement, tight 
junction and cell growth, which implies that cancer cells of patients with PTEN mutation might have greater mobility and migration.

In protein-protein interaction analysis, we identified the top 10 genes as the hub genes. GNG13, which encodes G Protein Subunit Gamma 13, functions as signal transducer for the 7-transmembrane-helix G proteincoupled receptors [31]. GO annotations related to this gene include obsolete signal transducer activity and Gprotein beta-subunit binding. In previous studies, the dysregulation of GNG13 was only found to be involved in the disease program of breast cancer [32]. Perhaps in the future, in-depth research will unearth the important role of this hub gene in prostate cancer. Other hub genes include ACTN2, POTEE, ACTA1, MYH6, MYH3, MYH7, MYL1, TNNC1 and TNNC2. One or several of the genes above might become new targets for prostate cancer research in the future.

Our study contained one limitation. In our study, we conducted a preliminary research on the effects of PTEN mutation in the progression and prognosis of prostate cancer. The mechanism and validation of PTEN mutations in prostate cancer still need further research in clinical and molecular biology experiments.

\section{Conclusions}

This study found out the main pathways and hub genes associated with PTEN mutation in prostate cancer. PTEN mutation is associated with advanced disease progression and the poorer prognosis of prostate cancer. Our results may facilitate the identification of PTEN mutation as a subgoup to improve prostate cancer prognosis prediction and develop therapeutic strategies.

\section{Supplementary information}

Supplementary information accompanies this paper at https://doi.org/10. 1186/s12881-019-0923-7.

Additional file 1: Fig. S1. Heat map of the top 100 differentially expressed genes. Red: up-regulation; purple: down-regulation.

Additional file 2. Hub genes list in protein-protein interaction analysis.

\section{Abbreviations}

DAVID: Database for annotation, visualization and integrated discovery; DEG: Differentially expressed gene; GEO: Gene Expression Omnibus": GO: Gene ontology; GSEA: Gene set enrichment analysis;" KEGG: Kyoto Encyclopedia of Genes and Genomes; MCODE: Molecular Complex Detection; PPI: Protein-protein interaction;; PTEN: Phosphatase and Tensin Homolog;" RNA-Seq: RNA sequencing; "STRING: Search Tool for the Retrieval of Interacting Genes/Proteins; TCGA: The Cancer Genome Atlas

\section{Acknowledgements}

Not applicable.

\section{Authors' contributions}

JQW: conception and design, obtaining funding and drafting the manuscript; JS and SGL: acquisition and analysis of the data and drafting the manuscript;
FW and CF: statistical analysis and technical support. All authors have read and approved the manuscript, and ensure that this is the case.

\section{Funding}

The authors disclosed receipt of the following financial support for the research, authorship, and/or publication of this article: This study was supported by National Natural Science Foundation of China (grant no. 81802565); Natural Science Foundation of Jiangsu Province (grant no. BK20180216); Key Project of the Scientific Research Project of Nanjing Medical University Affiliated Suzhou Hospital (grant no. szslyy2017005). All the financial support above support us mainly include downloading relevant data, obtaining database support, analyzing data, purchasing relevant analysis software and related researcher labor subsidies.

\section{Availability of data and materials}

Not applicable.

Ethics approval and consent to participate

Not applicable.

\section{Consent for publication}

Not applicable.

\section{Competing interests}

The author(s) declared no potential conflicts of interest with respect to the research, authorship, and/or publication of this article.

Received: 2 April 2019 Accepted: 12 November 2019

Published online: 02 December 2019

\section{References}

1. Siegel RL, Miller KD, Jemal A. Cancer statistics, 2016. CA Cancer J Clin. 2016; 66(1):7-30 Epub 2016/01/09.

2. Barbieri CE, Baca SC, Lawrence MS, Demichelis F, Blattner M, Theurillat JP, et al. Exome sequencing identifies recurrent SPOP, FOXA1 and MED12 mutations in prostate cancer. Nat Genet. 2012;44(6):685-9 Epub 2012/05/23.

3. Baca SC, Prandi D, Lawrence MS, Mosquera JM, Romanel A, Drier Y, et al. Punctuated evolution of prostate cancer genomes. Cell. 2013;153(3):666-77 Epub 2013/04/30.

4. Cooper CS, Eeles R, Wedge DC, Van Loo P, Gundem G, Alexandrov LB, et al. Analysis of the genetic phylogeny of multifocal prostate cancer identifies multiple independent clonal expansions in neoplastic and morphologically normal prostate tissue. Nat Genet. 2015;47(4):367-72 Epub 2015/03/03.

5. Taylor BS, Schultz N, Hieronymus H, Gopalan A, Xiao Y, Carver BS, et al. Integrative genomic profiling of human prostate cancer. Cancer Cell. 2010; 18(1):11-22 Epub 2010/06/29.

6. Wang S, Gao J, Lei Q, Rozengurt N, Pritchard C, Jiao J, et al. Prostate-specific deletion of the murine Pten tumor suppressor gene leads to metastatic prostate cancer. Cancer Cell. 2003;4(3):209-21 Epub 2003/10/03.

7. Robinson D, Van Allen EM, Wu YM, Schultz N, Lonigro RJ, Mosquera JM, et al. Integrative clinical genomics of advanced prostate Cancer. Cell. 2015; 162(2):454 Epub 2015/07/16.

8. Maehama T, Taylor GS, Dixon JE. PTEN and myotubularin: novel phosphoinositide phosphatases. Annu Rev Biochem. 2001;70:247-79 Epub 2001/06/08

9. Freeman DJ, Li AG, Wei G, Li HH, Kertesz N, Lesche R, et al. PTEN tumor suppressor regulates p53 protein levels and activity through phosphatasedependent and -independent mechanisms. Cancer Cell. 2003;3(2):117-30 Epub 2003/03/07.

10. Denmeade SR, Isaacs JT. A history of prostate cancer treatment. Nat Rev Cancer. 2002;2(5):389-96 Epub 2002/06/05.

11. Cerami E, Gao J, Dogrusoz U, Gross BE, Sumer SO, Aksoy BA, et al. The cBio cancer genomics portal: an open platform for exploring multidimensional cancer genomics data. Cancer discovery. 2012;2(5):401-4 Epub 2012/05/17.

12. Subramanian A, Tamayo P, Mootha VK, Mukherjee S, Ebert BL, Gillette MA, et al. Gene set enrichment analysis: a knowledge-based approach for interpreting genome-wide expression profiles. Proc Natl Acad Sci U S A. 2005;102(43):15545-50 Epub 2005/10/04.

13. Mootha VK, Lindgren CM, Eriksson KF, Subramanian A, Sihag S, Lehar J, et al. PGC-1alpha-responsive genes involved in oxidative phosphorylation are 
coordinately downregulated in human diabetes. Nat Genet. 2003;34(3):26773 Epub 2003/06/17.

14. Robinson MD, McCarthy DJ, Smyth GK. edgeR: a bioconductor package for differential expression analysis of digital gene expression data. Bioinformatics. 2010;26(1):139-40 Epub 2009/11/17.

15. McCarthy DJ, Chen Y, Smyth GK. Differential expression analysis of multifactor RNA-Seq experiments with respect to biological variation. Nucleic Acids Res. 2012;40(10):4288-97 Epub 2012/01/31.

16. Szklarczyk D, Franceschini A, Wyder S, Forslund K, Heller D, Huerta-Cepas J, et al. STRING V10: protein-protein interaction networks, integrated over the tree of life. Nucleic Acids Res. 2015;43(Database issue):D447-52 Epub 2014/10/30

17. Bader $\mathrm{GD}$, Hogue $\mathrm{CW}$. An automated method for finding molecular complexes in large protein interaction networks. BMC bioinformatics. 2003, 4:2 Epub 2003/01/15.

18. Fan C, Zhao C, Wang F, Li S, Wang J. Significance of PTEN mutation in cellular process, prognosis, and drug selection in clear cell renal cell carcinoma. Front Oncol. 2019;9:357 Epub 2019/05/30.

19. Huang R, Liao X, Li Q. Identification of key pathways and genes in TP53 mutation acute myeloid leukemia: evidence from bioinformatics analysis. OncoTargets and therapy. 2018;11:163-73 Epub 2018/01/19.

20. Salmena L, Carracedo A, Pandolfi PP. Tenets of PTEN tumor suppression. Cell. 2008:133(3):403-14 Epub 2008/05/06.

21. Steelman LS, Bertrand FE, McCubrey JA. The complexity of PTEN: mutation marker and potential target for therapeutic intervention. Expert Opin Ther Targets. 2004;8(6):537-50 Epub 2004/12/09.

22. Wang Y, Romigh $T$, He X, Tan MH, Orloff MS, Silverman RH, et al. Differential regulation of PTEN expression by androgen receptor in prostate and breast cancers. Oncogene. 2011:30(42):4327-38 Epub 2011/05/03.

23. Seront E, Pinto A, Bouzin C, Bertrand L, Machiels JP, Feron O. PTEN deficiency is associated with reduced sensitivity to MTOR inhibitor in human bladder cancer through the unhampered feedback loop driving PI3K/Akt activation. Br J Cancer. 2013;109(6):1586-92 Epub 2013/08/31.

24. Bruni P, Boccia A, Baldassarre G, Trapasso F, Santoro M, Chiappetta G, et al. PTEN expression is reduced in a subset of sporadic thyroid carcinomas: evidence that PTEN-growth suppressing activity in thyroid cancer cells mediated by p27kip1. Oncogene. 2000;19(28):3146-55 Epub 2000/08/03.

25. Sidaway P. Prostate cancer: targeting lipid metabolism. Nature reviews Urology. 2017;14(4):196 Epub 2017/03/02.

26. Wu X, Daniels G, Lee P, Monaco ME. Lipid metabolism in prostate cancer. American journal of clinical and experimental urology. 2014;2(2):111-20 Epub 2014/11/07.

27. Grasso CS, Wu YM, Robinson DR, Cao X, Dhanasekaran SM, Khan AP, et al. The mutational landscape of lethal castration-resistant prostate cancer. Nature. 2012:487(7406):239-43 Epub 2012/06/23.

28. Beltran H, Yelensky R, Frampton GM, Park K, Downing SR, MacDonald TY, et al. Targeted next-generation sequencing of advanced prostate cancer identifies potential therapeutic targets and disease heterogeneity. Eur Urol. 2013:63(5):920-6 Epub 2012/09/18

29. Mateo J, Carreira S, Sandhu S, Miranda S, Mossop H, Perez-Lopez R, et al. DNA-repair defects and Olaparib in metastatic prostate Cancer. N Engl J Med. 2015;373(18):1697-708 Epub 2015/10/29.

30. Martin SK, Kyprianou N. Exploitation of the androgen receptor to overcome Taxane resistance in advanced prostate Cancer. Adv Cancer Res. 2015;127: 123-58 Epub 2015/06/22.

31. Fujino A, Pieretti-Vanmarcke R, Wong A, Donahoe PK, Arango NA. Sexual dimorphism of G-protein subunit Gng13 expression in the cortical region of the developing mouse ovary. Developmental dynamics : an official publication of the American Association of Anatomists. 2007;236(7):1991-6 Epub 2007/05/15.

32. Fu Y, Kadioglu O, Wiench B, Wei Z, Wang W, Luo M, et al. Activity of the antiestrogenic cajanin stilbene acid towards breast cancer. J Nutr Biochem. 2015;26(11):1273-82 Epub 2015/09/15.

\section{Publisher's Note}

Springer Nature remains neutral with regard to jurisdictional claims in published maps and institutional affiliations.

\section{Ready to submit your research? Choose BMC and benefit from:}

- fast, convenient online submission

- thorough peer review by experienced researchers in your field

- rapid publication on acceptance

- support for research data, including large and complex data types

- gold Open Access which fosters wider collaboration and increased citations

- maximum visibility for your research: over $100 \mathrm{M}$ website views per year

At $\mathrm{BMC}$, research is always in progress.

Learn more biomedcentral.com/submissions 